\title{
La contratación de la Misión Klein- Saks por Chile (1955): el papel de Estados Unidos y los organismos financieros internacionales ${ }^{1}$
}

\author{
The hiring of the Klein-Saks Mission by Chile (1955): \\ the role of the United States and international financial organizations \\ Daniel Ahumada Benítez \\ Universidad de Santiago de Chile, Chile. $\measuredangle$ daniel.ahumada@usach.cl \\ [orcid.org/0000-0001-6793-1094]
}

\section{RESUMEN}

El objetivo de este trabajo es examinar la participación e importancia que tuvieron los organismos financieros internacionales y el gobierno estadounidense en el proceso de contratación de la Misión Klein-Saks por parte de la administración de Carlos Ibáñez del Campo para mediados de 1955. Se plantea que el gobierno estadounidense y las organizaciones financieras internacionales presionaron a la administración de Ibáñez, desde 1954, para que solucionara la situación de la inflación y contratara expertos para ello. Agregar que desde Estados Unidos no hubo una recomendación explícita ni directa para que Chile contratara específicamente a la firma Klein-Saks.

PALABRAS CLAVE: Misión Klein-Saks, organismos financieros internacionales, Estados Unidos, Carlos Ibáñez del Campo.

\section{ABSTRACT}

This paper aim to examine the participation and importance of the U.S. government and the international financial organizations in the hiring process of the Klein-Saks Mission by the administration of Carlos Ibáñez del Campo in the mid of 1955. It is argued that the government of the United States and the international financial organizations pressured the administration of Ibáñez, since at least 1954, to solve the inflation situation y hire

1 Este trabajo es producto de la investigación realizada durante el proceso de tesis para obtener el grado de Magíster en Estudios Internacionales (2018), titulada “Alineamiento, pragmatismo e interés: la política exterior del gobierno de Carlos Ibáñez del Campo hacia Estados Unidos, 1952-1958”. 
experts for it. Besides, from the United States authorities there wasn't an explicit or direct recommendation to Chile to hire specifically the Klein-Saks firm.

KEY WORDS: Klein-Saks Mission, international financial organizations, United States, Carlos Ibáñez del Campo.

\section{INTRODUCCIÓN}

La Misión Klein-Saks fue un grupo de expertos estadounidenses que prestaron servicios al gobierno de Carlos Ibáñez del Campo (1952-1958), entre mediados de 1955 hasta mediados de 1958, con el propósito de hacer frente a la situación económica del país (principalmente caracterizada por una alta inflación), recomendando y ayudando en la aplicación de políticas económicas. Un tema recurrente en la literatura se refiere a las razones que llevan a explicar la contratación de expertos extranjeros para asesorar al gobierno chileno y por qué específicamente se contactó a la consultora Klein-Saks. Hay consenso en plantear que ello respondió a una medida para reducir la elevada inflación, especialmente para 1954-1955. Sin embargo, existen varias perspectivas que complejizan o complementan la respuesta.

Algunos autores destacan que, a la delicada situación económica durante los años de Ibáñez, se sumó un contexto de crisis política, donde el gobierno de turno tuvo dificultades para formar coaliciones políticas y, además, se generó una importante movilización social, entonces se hacía necesario solucionar el problema de la inflación para no agudizar aún más la situación del país (Hirschman, 1964, pp. 219-239; Pinto, 1964, p. 180; Collier \& Sater, 1999, p. 241; Correa et al. 2001, pp. 202-203; Couyoumdjian, 2011). También, se ha planteado que el propósito de la contratación de la Misión Klein-Saks estuvo en posibilitar un acercamiento entre el gobierno de Ibáñez y los partidos de derecha, el Liberal y Conservador, para concretar una alianza política (Ffrench-Davis, 1973, pp. 2526; Couyoumdjian, 2011, pp. 85-86; Edwards, 2011, pp. 144-146; Gil, 1969, p. 209). Otros autores agregan que, en aquel momento histórico, se pensaba que una misión financiera compuesta por "técnicos extranjeros" y “alejados de la contingencia política” de Chile entregaría la credibilidad necesaria ante diferentes actores nacionales e internacionales para que el gobierno pudiera impulsar planes de estabilización económica (Kofas, 1999, pp. 366-367), algunos destacando que especialmente se esperaba el apoyo de la derecha política con la Misión (Edwards, 2011) y otros que entregaría credibilidad ante el Fondo Monetario Internacional (Sierra, Benavente \& Osorio, 1967, pp. 40-41). Y se han destacado las recomendaciones y presiones realizadas por el gobierno estadounidense, FMI y el Banco Internacional de Reconstrucción y Fomento (BIRF) sobre el gobierno de Ibáñez para que solucionaran la situación inflacionaria (Kofas, 1999), con algunos autores enfocándose en la acción del FMI sobre la administración de Ibáñez (Sierra et al. 1967, pp. 38-49) y otros aludiendo a las presiones del gobierno estadounidense y FMI (Wilhelmy, 1979, pp. 458-459). 
Para responder al porqué se establecieron vínculos con la firma Klein-Saks, se ha hecho referencia a la importancia que tuvo Agustín Edwards Budge, director de El Mercurio para entonces, quien promocionó y convenció al gobierno de Carlos Ibáñez del Campo para contratar a los expertos de la firma Klein-Saks ; (Würth, 1958, p. 336; Hirschman, 1964, p. 230; Correa, 1985; Correa et al. 2001, pp. 202-203; Hove, 2009, p. 198; Couyoumdjian, 2011, pp. 75-79). Otros lo explican por la posibilidad de obtener con mayor facilidad la ayuda y préstamos internacionales, debido a los vínculos y contactos de la firma Klein-Saks con actores del gobierno estadounidense y de los organismos financieros internacionales (Hirschman, 1964, p. 230; Sierra et al. 1967, p. 37; Ffrench Davis, 1973, pp. 25-26; Sater, 1990, p. 127; Collier \& Sater, 1999, p. 241; Correa et al. 2000, pp. 202-203; Hove, 2009, pp. 198-199). Por último, se ha escrito que se escogió a la firma Klein-Saks debido a que era auspiciada por la Minera Anaconda, debiéndole Ibáñez favores a esa empresa por la ayuda recibida de ella en el proceso de aprobación de la ley del "nuevo trato" del cobre de 1954 (Kofas, 1999, p. 367).

A partir de todas estas perspectivas nombradas, hay que considerar que ellas no son excluyentes entre sí, lo cual implica establecer que la adquisición de los servicios de la Misión Klein-Saks por parte del gobierno chileno en 1955 respondió a una multicausalidad. Además, entre la variedad de factores o circunstancias referidas por la bibliografía académica sobre el convenio con la Misión, se observa que la importancia de actores externos, como el gobierno estadounidense y los organismos financieros internacionales, para explicar la contratación de los expertos extranjeros ha sido tratada sin mayor profundidad. Se puede destacar el trabajo de Kofas (1999), quien estudia los contactos entre el gobierno de Ibáñez con el Departamento de Estado, el FMI y el BIRF a lo largo de 1952-1958. Este autor alude a las conversaciones que hubo sobre el tema de la inflación, sin embargo, no entra en mayor detalle sobre la contratación de la Misión Klein-Saks. Por su parte, Sierra et al. (1967) desarrollan sobre el papel de la influencia y presiones del FMI para que el gobierno de Ibáñez solucionara el problema de la alta inflación, pero centrándose solo en la acción del Fondo Monetario Internacional y sin referenciar fuentes primarias específicas que respalden muchas de sus afirmaciones.

En este trabajo se quiere profundizar aún más en la importancia de los actores internacionales (el gobierno estadounidense y organismos financieros internacionales), entendiendo que la acción realizada por ellos es una variable que ayuda a explicar el convenio con la Misión Klein-Saks de 1955. ¿Cuál fue la postura del gobierno estadounidense y los organismos financieros internacionales ante la adopción por parte de Chile de la Misión Klein Saks? ¿Intervinieron en el proceso de contratación de la Misión? ¿Incentivaron al gobierno de Ibáñez para adquirir sus servicios? Por tanto, el objetivo de este trabajo es examinar la participación del gobierno estadounidense y de los organismos financieros internacionales en el proceso que llevó a la contratación de la Misión Klein-Saks por parte de Chile en 1955. Puntualizar que en este escrito la denominación "organismos financieros internacionales" hace referencia a tres específicamente: el Fondo Monetario Internacional (FMI), el Banco 
Internacional de Reconstrucción y Fomento (BIRF) y el Banco de Exportación e Importación de Estados Unidos (Eximbank). Si bien el último de los nombrados es un organismo gubernamental y no tiene el mismo carácter que los otros dos, para facilitar el análisis se agrupan estas tres instituciones bajo un mismo concepto.

Las fuentes primarias utilizadas son diplomáticas, tanto de Chile como de Estados Unidos. Desde el punto de vista chileno se revisó la documentación del Archivo Histórico del Ministerio de Relaciones Exteriores (AMRREE). Desde la perspectiva estadounidense se utilizaron los archivos diplomáticos de la serie Foreign Relations of the United States que tratan sobre las relaciones entre Chile y Estados Unidos, los que están presentes en la página web del Departamento de Estado, específicamente en sección Office of The Historian².

Se plantea que: 1) el gobierno estadounidense y los organismos financieros internacionales tomaron una postura, desde al menos 1954, de presionar a la administración de Carlos Ibáñez del Campo para que tuviera como prioridad solucionar el problema de la inflación, lo cual evolucionó para 1955 a una presión para que contratara una misión de expertos internacionales que les ayudara para estabilizar la economía. 2) Si bien desde EE.UU. se mostraron conformes con la posibilidad de que Chile solicitara los servicios de la firma Klein-Saks en 1955, no la recomendaron ni exigieron su contratación de manera directa o explícita al gobierno de Ibáñez.

Este trabajo se puede inscribir dentro de la rama disciplinaria de la Historia Económica, ya que trata un tema vinculado a la política económica de un país en el pasado. Sin embargo, también tiene elementos que lo asocian a la subdisciplina de la Historia de las Relaciones Internacionales, la cual entrega luces sobre cómo abordar el problema de investigación de este escrito. Pereira y Neila (2003) definen a esta última subdisciplina nombrada como: "el estudio científico y global de las relaciones históricas que se han desarrollado entre los hombres, los Estados y las colectividades supranacionales en el seno de la sociedad internacional” (p. 14), de lo cual se desprende que las investigaciones desde esta perspectiva vinculan el sistema internacional y marcos estructurales globales con las relaciones específicas entre los sujetos históricos. En una línea parecida y de manera algo más precisa, Joaquín Fermandois (2009) plantea que la historia de las relaciones internacionales es estudiar un fenómeno internacional desde un punto de vista historiográfico, considerando tres dimensiones: el sistema internacional, la sociedad interna de los Estados y las relaciones internacionales específicas desarrolladas por los sujetos.

Un primer apartado de este artículo se refiere a la contextualización de la Guerra Fría. Luego se abordan los vínculos entre Chile y Estados Unidos desde el punto de vista de las relaciones comerciales en contexto de Guerra Fría. Tercero, sobre las condiciones económicas y políticas internas de Chile durante los años de Ibáñez. Y un cuarto apartado desarrolla la postura y participación del gobierno estadounidense y las organizaciones financieras internacionales en el proceso de contratación de la Misión Klein-Saks, a partir de la revisión de los documentos diplomáticos chilenos y de EE.UU.

Dichos documentos se encuentran en: https://history.state.gov/historicaldocuments/frus1955-57v07/ch11. 


\section{LA SITUACIÓN INTERNACIONAL DURANTE LA GUERRA FRÍA}

Estados Unidos se posicionó como la principal potencia del sistema internacional al término de la Segunda Guerra Mundial, adquiriendo el prestigio de ser una de las naciones vencedoras del conflicto, poseedora de una gran fuerza militar, con un potencial económico destacado, con la capacidad de impulsar la creación de instituciones internacionales (como las Naciones Unidas) y con el poder necesario para configurar las reglas bajo las que se enmarcarían las relaciones internacionales. Sin embargo, la Unión Soviética se transformó en la otra gran potencia al término de la Segunda Guerra Mundial, representando esta nación un modelo político y de desarrollo alternativo al de EE.UU. Estas dos naciones fueron las dos principales protagonistas del periodo conocido como la Guerra Fría, tendiendo conflictuar y competir entre ellas.

Bajo ese marco internacional, para Hobsbawm (1999, pp. 231-232) los países del Tercer Mundo tendieron a tener mayor afinidad por el llamado "bando occidental", ya que, a pesar de la poca "simpatía" por Estados Unidos, en general no eran comunistas, siendo más bien anticomunistas en política interior y "no alineados" en política exterior. Latinoamérica no estuvo exenta de tener una mayor inclinación hacia Estados Unidos.

La Guerra Fría para Peter Smith (1996, p. 6) fue un momento histórico que estableció determinados marcos bajo los cuales se desarrollaron las relaciones entre América Latina y Estados Unidos, siendo un conflicto que tuvo como características: 1) la rivalidad entre la Unión Soviética y los estadounidenses, la que se desarrolló a una escala planetaria y fue intensificada por la tenencia de armas nucleares por ambos bandos. 2) Se gestó un cambio de relación entre EE.UU. y Latinoamérica, debido a que las regiones del Tercer Mundo fueron un campo de batalla entre las potencias en lucha, llegando a considerarse la influencia sobre esas partes del mundo como el premio o recompensa de esa pugna. Para Smith la "seguridad nacional" se posicionó en la prioridad dentro de la agenda internacional estadounidense, lo cual implicaba enfrentarse a la URSS por el Tercer Mundo con el fin de mantener dentro de su órbita de influencia a la mayor cantidad de naciones. Y 3) la existencia de una política de “contención" en contra del comunismo, desarrollada en los inicios de la Guerra Fría, con la que Estados Unidos se consolidó y extendió en mayor grado su supremacía en las regiones bajo su órbita, aplicando políticas para mantener su hegemonía.

Estados Unidos generó una serie de políticas de ayuda económica hacia diferentes países del mundo como una forma de apoyarlos en el camino hacia el desarrollo y, de esa manera, evitar la "infiltración comunista", lo cual se ideó a partir de la política de "contención" desarrollada por George F. Kennan en 1947 y por la "Doctrina Truman" expuesta por Harry Truman. Éstas respondieron al propósito de resguardar la seguridad nacional estadounidense y evitar una mayor influencia de la URSS en el mundo. América Latina también fue objeto de la ayuda estadounidense. Louis Halle, funcionario del Departamento de Estado, escribió en 1950 para la revista Foreign Affairs, bajo el seudónimo de "Y", un artículo en que propuso que Estados 
Unidos debía apoyar a los países americanos a consolidar sus democracias, debido a que aún no eran "maduras", y para ello era necesario: 1) enfrentar las desigualdades y problemas sociales de todas las naciones americanas, con acciones de cooperación y trabajo conjunto de los países del continente, siendo importante la participación en las instancias internacionales de coordinación; y 2) generar desarrollo económico en los países latinoamericanos como un objetivo necesario para consolidar la democracia en todo el continente, y para ello era necesario la acción de instituciones como el Eximbank y el BIRF (Y [Louis Halle], 1950, p. 576).

Chile tendió a un alineamiento con Estados Unidos durante la Guerra Fría y también fue objeto de la política estadounidense de apoyar a las naciones del Tercer Mundo para que alcanzaran el desarrollo y así evitar la "expansión del comunismo”.

\section{LOS VÍNCULOS ECONÓMICOS DE CHILE CON ESTADOS UNIDOS}

Una situación fundamental a considerar es la importancia de las relaciones económicas entre las naciones latinoamericanas con EE.UU. durante la Guerra Fría. Chile no fue ajeno a ello, siendo el nombrado país su principal socio comercial, su más importante comprador de materias primas y una fuente significativa de inversión extranjera y ayuda monetaria.

Centrándose específicamente en lo que fue la presidencia de Carlos Ibáñez del Campo, se puede observar la importancia de los vínculos económicos con Estados Unidos. Un informe elaborado en el Ministerio de Relaciones Exteriores, bajo el título de "Breve análisis de las relaciones comerciales entre Chile y Estados Unidos" y fechado para el 16 de noviembre de 1957, que trata sobre las relaciones comerciales entre las dos naciones entre 1952 y 1956, constata la posición de EE.UU. como principal socio económico de Chile ${ }^{3}$. Sin entrar en una descripción detallada del informe, en el mismo documento se resumen algunas de las principales condiciones del intercambio entre Chile y Estados Unidos:

1) El intercambio chileno-norteamericano representa alrededor del $50 \%$ del intercambio total del país;

(...)

3) las exportaciones chilenas a los Estados Unidos corresponden en su gran mayoría a productos de la minería (...);

4) Las importaciones comprendieron principalmente bienes de capital, materias primas, productos agropecuarios (por Convenios de Excedentes Agrícolas) y algunas manufacturas de hierro y acero, en el mismo orden (...). (AMRREE, Vol. 4639, Breve análisis de las relaciones comerciales entre Chile y Estados Unidos, 16 de noviembre de 1957).

Si bien los datos que entrega el informe no abarcan el gobierno completo de Carlos Ibáñez, de igual forma se puede considerar representativos de lo que fue el intercambio comercial entre los dos países entre 1952-1958, considerando que para 1957 y 1958 no fueron años en que ocurrió algo que modificara sustancialmente las características de los vínculos económicos entre las dos naciones. 
De esta cita se refleja que al interior del gobierno de Ibáñez se tuvo conciencia de la importancia del intercambio comercial con Estados Unidos, el que representaba alrededor del $50 \%$ del total realizado por Chile, y de la composición de las exportaciones (principalmente productos primarios de la minería) y de las importaciones (bienes mayormente elaborados).

A esto hay que agregar la ayuda económica que Estados Unidos entregaba a otros países, como Chile. Desde la perspectiva de Fermandois (2002, 2005) y Fermandois, Bustos y Schneuer (2009), durante el periodo que abarcó desde mediados de la década de 1940 hasta 1970, estuvo muy difundida la lógica del "subsidio", la que consistía en que el mundo político chileno consideraba que EE.UU. tenía la obligación de apoyar a Chile en su camino hacia el desarrollo económico, ya fuese mediante la compra de materias primas, la facilitación de ayuda económica o la entrega de algún beneficio. Esto implicó que la clase política chilena estuvo predispuesta a recibir ayuda monetaria o préstamos internacionales. Efectivamente, Chile aceptó la asistencia de Estados Unidos, que fue en ayuda económica directa, créditos e incluso aportes monetarios para gasto militar. Y a ello hay que sumar las constantes gestiones realizadas por los gobiernos chilenos para adquirir créditos de organismos financieros internacionales. De acuerdo a Muñoz y Portales (1987) la ayuda internacional hacia Chile durante la Guerra Fría fue importante:

Si se examinan las estadísticas de los programas de donaciones y créditos norteamericanos hacia América Latina se puede comprobar la alta prioridad asignada a Chile, país que, salvo periodos extraordinarios, recibió donaciones y créditos en una proporción muy superior a su participación en el producto y en la población de América Latina (Muñoz \& Portales, 1987, p. 46).

Entonces, durante los años de Ibáñez, y en general durante el periodo de la Guerra Fría, los vínculos económicos con Estados Unidos fueron importantes, tanto desde el punto de vista comercial como de la asistencia económica recibida.

\section{LA SITUACIÓN INTERNA DURANTE LOS AÑOS DE CARLOS IBÁÑEZ DEL CAMPO}

Comúnmente se alude a que los años del gobierno de Carlos Ibáñez del Campo fueron complejos, tanto desde un punto de vista económico como político. Como elemento característico de este periodo se destaca el alza inflacionaria que se vivió, donde se alcanzaron niveles no vistos hasta ese momento en la historia de Chile.

A partir de los datos de la Tabla $\mathrm{N}^{\circ} 1$ se observa que, si bien la inflación ya venía con la tendencia de ser elevada desde antes de 1952 (dentro del rango de los dos dígitos

4 Hay que acotar que la inflación puede ser considerada como un problema presente en Chile desde fines del siglo XIX, incluso llegando a escribir Hirschman en 1964: “(...) el locus classicus de la inflación en la América Latina es Chile, que ha venido atravesando un proceso inflacionario sumamente prolongado y virulento." (Hirschman, 1964, p. 182). 
Tabla 1. Tasa de inflación 1947-1958.

\begin{tabular}{cccc}
\hline Año & Tasa de inflación & Año & Tasa de inflación \\
\hline 1947 & 23,1 & 1953 & 56,6 \\
1948 & 16,8 & 1954 & 71 \\
1949 & 20,4 & 1955 & 84 \\
1950 & 16,7 & 1956 & 37,8 \\
1951 & 22,8 & 1957 & 17,1 \\
1952 & 12 & 1958 & 32,5 \\
\hline
\end{tabular}

Datos presentes en Edwards (2011, p. 105), a partir de estadísticas del FMI.

porcentuales), durante los años del gobierno de Ibáñez fue especialmente alta. A partir de 1953 comenzó un aumento importante en el alza de los precios, pasando del 12\% en 1952 a un 56.6\% en 1953 y llegando al 84\% en 1955. Aunque desde 1956 comenzó una disminución de la inflación, de igual forma continuó dentro del margen de los dos dígitos.

A la difícil situación económica, hay que agregar que Ibáñez llegó a la presidencia en 1952 mediante un apoyo político muy heterogéneo, abarcando desde partidos de izquierda hasta nacionalistas. Una vez en el poder, esto significó dificultades para llevar la conducción del gobierno, con una falta de dirección clara y cambios de tendencia en las políticas realizadas. Para Tomás Moulian (1986) la administración de Ibáñez tuvo tres etapas definidas: 1) un "periodo populista", desde inicios de su mandato hasta mediados de 1955, donde coexistieron en la coalición gobernante grupos políticos de diferentes tendencias, 2) un "vuelco a la derecha", desde mediados de 1955 hasta inicios de 1958, el cual estuvo caracterizado por la vinculación del gobierno con los partidos tradicionales de derecha y donde se contrató a la Misión KleinSaks, y 3) un "viraje a la izquierda” para el último año de Ibáñez en el poder, momento en que el gobierno colaboró con partidos de izquierda y centro para impulsar una serie de medidas, entre las que estuvieron una reforma electoral y la derogación de la llamada "ley maldita". Como se puede concluir, Ibáñez tuvo dificultades para formar y mantener una coalición política estable, lo que significó cambios constantes de aliados y recurrentes problemas políticos.

Por otro lado, además de la situación política difícil, los problemas económicos, con continuas alzas de precios y pérdida del poder adquisitivo de los salarios, llevaron a un creciente descontento y agudizaron la movilización social. Esto fue patente en 1955, describiendo Hirschman (1964) de la siguiente manera el contexto:

Tras los enormes aumentos de sueldos del 60 por 100 en enero de 1955, la inflación cobró toda su potencia en los primeros meses del año. En el primer semestre hubo tres ministros de Hacienda, y se multiplicaron rápidamente las manifestaciones de desintegración política y social. Las huelgas se hicieron más frecuentes y se extendieron a servicios tan esenciales como los transportes y hospitales (...). En julio, la Confederación Única de Trabajadores Chilenos (...) declaró una huelga general, a la que siguieron otras graves huelgas en agosto (Hirschman, 1964, p. 227). 
Bajo el momento del país descrito por la cita (de inflación en aumento, fatal de una conducción clara de política económica en el gobierno y una agudización de la protesta social) fue que se firmó el convenio con la consultora estadounidense Klein-Saks en julio de 1955. Aunque, hay que destacar que, antes de la Misión Klein-Saks, el gobierno de Ibáñez tuvo propuestas que buscaron el control de la inflación, específicamente bajo los ministros de Hacienda Felipe Herrera en 1953 y Jorge Prat en 1954 . La falta de decisión política dentro de la administración de Ibáñez pudo ser un factor que explica el que no progresaran esos programas antiinflacionarios (Couyoumdjian, 2011, pp. 71-72).

\section{LA POSTURA ESTADOUNIDENSE ANTE LA MISIÓN KLEIN-SAKS}

Los altos niveles de inflación en Chile para el momento de 1954-1955 no solo fueron tema de preocupación y análisis al interior del país, también las autoridades estadounidenses y organismos financieros internacionales estuvieron pendientes al respecto. Desde el gobierno norteamericano establecieron formas de apoyar a la administración de Ibáñez ante la difícil situación económica. En un documento preparado para un grupo de trabajo del Operations Coordinating Board ${ }^{6}$, fechado el 17 de diciembre de 1954, plantearon las principales líneas de acción que debía seguir el gobierno de Dwight Eisenhower para apoyar a Chile. Partieron de la base que solo los chilenos podían llevar a cabo las reformas necesarias para estabilizar su economía y que la ayuda estadounidense no podía ser efectiva a menos que el gobierno de Ibáñez iniciara la aplicación de medidas que condujeran al control de la inflación (Foreign Relations of the United States, 1952-1954, Vol. IV, Documento 260, 17 de diciembre de 1954)7.

Entre las recomendaciones que estableció el aludido informe estuvo que: el FMI debía apoyar y presionar para que Chile generase reformas macroeconómicas y que el Eximbank y el BIRF debían entregar la ayuda monetaria necesaria si el gobierno chileno aplicaba un programa de reformas promisorio (Foreign Relations of the United States..., Documento 260, 17 de diciembre de 1954). Otras recomendaciones fueron que la Embajada estadounidense en Santiago debía tener contacto con personajes claves de Chile y convencerlos para que se tomaran medidas sobre la inflación y la estabilización de la economía y, además, recomendar la posibilidad de crear un Anti-Inflation Advisory Comittees al gobierno chileno (Foreign Relations of the United States..., Documento 260, 17 de diciembre de 1954).

Mediante la revisión de documentación diplomática, se puede plantear que las recomendaciones nombradas efectivamente se llevaron a la práctica, específicamente

Una descripción de estos dos planes se puede ver en Edwards (2011, pp. 118-122) y Hirschman (1964, pp. 219227).

6 El Operations Coordinating Board (OCB) fue organismo estadounidense, creado durante el gobierno de Eisenhower, encargado de coordinar las políticas de seguridad nacional de ese país.

7 El informe planteó: "The consensus of the Group is that only the Chileans can bring about the reforms necessary to stabilize and strengthen their economy and that U.S. aid cannot be effective unless the Chileans enact and implement the required reform measures". El documento se puede ver en: https://history.state.gov/ historicaldocuments/frus1952-54v04/d260. 
mediante la acción diplomática de la Embajada estadounidense en Chile y de los organismos financieros internacionales. Ello ya se observó en una reunión realizada por el Directorio Ejecutivo del Fondo Monetario Internacional para el 1 de diciembre de 1954, en que discutieron los resultados de un informe realizado por una Misión de técnicos del FMI que visitaron Chile durante 1954, quienes estudiaron la situación económica nacional y un plan de modificación al sistema cambiario que estaba proponiendo el gobierno de Ibáñez. La Embajada chilena en Washington envió el acta de dicha reunión del Directorio Ejecutivo del FMI al Ministerio de Relaciones Exteriores en Chile (AMRREE, Vol. 4077, Estrictamente Confidencial N ${ }^{\circ} 33-3,10$ de enero de 1955). El principal tema de discusión de la reunión fue la creciente inflación en Chile y la necesidad de que la administración de turno tuviera la prioridad de enfrentarla y, solo después de ello, pensar en otros asuntos. Uno de los asistentes, Frank A. Southard, fue el primero en poner el foco en el problema de la inflación y que la acción y opiniones del FMI debían ir en la dirección de tratar ese asunto antes que otros.

A su modo de ver [el de Southard], el aspecto más interesante del Plan de Rectificación Económica del Gobierno era el control de la inflación. (...) hacía necesarias las opiniones más francas del Fondo respecto del programa antiinflacionista. Estas eran más importantes que las opiniones del Fondo en el aspecto cambiario, debido a que el freno a la inflación era tan fundamental para el éxito de un programa cambiario. Le parecía decepcionante y conturbador que el Gobierno planeara no hace otra cosa en el primer año del programa bienal que mantener en 30 a 40 por ciento el aumento de los precios (AMRREE, Vol. 4077, "Fondo Monetario Internacional. Reunión del Directorio Ejecutivo. Acta del 1\% XII/1954”, p. 2).

Una de las críticas realizadas por parte de los participantes de la reunión, fue que pensaban que el gobierno chileno no proponía las medidas necesarias para que efectivamente disminuyera la inflación. Otro de los presentes en la reunión, Otto Donner, rebatió la idea de que, para superar los problemas, Chile debía principalmente incrementar su producción y productividad, diversificar su industria y aumentar las exportaciones. Más bien esperaba que se aplicaran otras políticas:

Dudaba [Donner] si era posible curar las dificultades monetarias básicas del país considerado solamente el aspecto físico de la economía. Mientras que simpatizaba con la intención del gobierno de frenar las presiones inflacionistas aumentado gradualmente la producción, no creía que eso podría cumplir toda la tarea. Se necesitaba un fuerte crédito anti-inflacionista y políticas fiscales. El hecho de que el gobierno está dispuesto a que los precios aumenten de un 30\% a un 40\% en un año parecía preparar el cuadro para una espiral inflacionista ascendente (AMRREE, Vol. 4077, "Fondo Monetario Internacional. Reunión del Directorio Ejecutivo. Acta del 1XXI/1954”, p. 7). 
Otro de los presentes, Viscount Harcourt, insistió en la necesidad de abordar la inflación y que el gobierno chileno debía hacer reales esfuerzos para enfrentarla, si es que quería seguir teniendo el apoyo del FMI:

Harcourt concordó en que la situación inflacionista era lo primero que debía abordarse y respecto de la que el Fondo debía dar consejos firmes y útiles a Chile. Creía que el Fondo debería llevar al ánimo de Chile que la situación requería medidas fiscales rigurosas dentro del país. Perturbaba observar que las autoridades chilenas esperaban que el control de la inflación tomaría dos años; ciertamente, la situación necesitaba un programa mucho más estricto que ese. En 1953 cuando Chile giró contra los recursos del Fondo, había un consenso definitivo de que se pondría en vigencia un plan definitivo que abarcara el control de la inflación. No obstante, el Fondo se encontraba actualmente frente al informe de que la situación inflacionista había empeorado y que se permitiría un aumento de 30 a 40\% en los precios. Claramente, si se autorizara a Chile para girar nuevamente contra los recursos del Fondo, este país debería hacer serios esfuerzos para controlar la inflación (...) estaba perturbado por la admisión de debilidad por parte de las autoridades chilenas, cuando ellas señalaron a la Misión del Secretariado que no podía, en el presente, seguir políticas fiscales y monetarias más estrictas (AMRREE, Vol. 4077, "Fondo Monetario Internacional. Reunión del Directorio Ejecutivo. Acta del 1\%/XII/1954”, p. 8).

También, a inicios de marzo de 1955, desde la Embajada chilena en Washington hicieron llegar al Ministerio de Relaciones Exteriores una carta enviada por el Presidente del Banco Internacional de Reconstrucción y Fomento, Eugene R. Black, dirigida al presidente de Chile. Dicha misiva fue una respuesta del BIRF ante la intención del gobierno de Ibáñez de implementar un "Programa de Desarrollo de la Agricultura y los Transportes en la República de Chile", argumentando dicha carta que era necesario primero solucionar el problema de la inflación y, una vez superada esa dificultad, empezar la implementación del plan de desarrollo de la agricultura, planteándole Black que:

En realidad recordará $U d$. Que nuestra carta de $1^{\circ}$ de diciembre 1952 con la que le enviamos el Informe de la Comisión conjunta del Banco y de la FAO, sobre "La Economía Agrícola de Chile", el señor Dodd y yo señalamos que los esfuerzos para modernizar y mejorar la producción agrícola de Chile no tendrían gran éxito a menos que se adoptaran medidas efectivas para detener la inflación que ha sufrido Chile en forma casi ininterrumpida durante los últimos veinte años (AMRREE, Vol. 4077, Confidencial N²32/17, 8 de marzo de 1955).

Además, Black dijo que desde el Banco Internacional de Reconstrucción y Fomento enviarían una misión a Chile con el propósito de actualizar sus antecedentes económicos y financieros: 
(...) he dado instrucciones a la Misión de que concentre su atención en esta etapa sobre las medidas que se han adoptado y aquellas que se proyectan, a fin de detener las fuerzas inflacionistas, para suministrar un financiamiento adecuado a los costos internos del programa y para volver a crear incentivos a las inversiones y a la producción en la agricultura y otros campos (AMRREE, Vol. 4077, Confidencial N²32/17, 8 de marzo de 1955).

Las dos situaciones nombradas (reunión del FMI y carta del BIRF) ejemplifican la postura de los organismos financieros internacionales de recomendar, e incluso presionar, para que la administración de Ibáñez realizara medidas para controlar la inflación como una primera tarea antes de cualquier otro plan de tipo económico o financiero. En el caso de la reunión con el FMI, aludieron a que el gobierno chileno no debía permitir un alza en porcentajes elevados de los precios a lo largo de un año, discutieron la posibilidad de generar cambios en la seguridad social para limitar sus beneficios, que se disminuyeran los costos administrativos del gobierno o que se evitara un aumento de los salarios, al mismo tiempo de un déficit presupuestario, todo esto con el fin de controlar los efectos inflacionarios. Además, a partir de la postura de Harcourt, se observa que se deslizó la idea de condicionar la facilitación de créditos del FMI a Chile, si es que no generaban acciones concretas para controlar la inflación. En el caso del diálogo con el BIRF, argumentaron la necesidad de controlar el alza de los precios, ya que, solo de esa manera, los programas de desarrollo y producción podían llegar a tener resultados positivos.

En un reporte elaborado por la Operations Coordinating Board sobre la situación de Chile, fechado para el 13 de abril de 1955, se constata que los estadounidenses continuaron con la postura de considerar que el gobierno de Ibáñez debía primero solucionar el problema de la inflación y, solo después de ello, podía EE.UU. entregar algún tipo de ayuda. En dicho documento, nombran que hasta ese momento la acción norteamericana había sido de incentivar a los chilenos para que tomaran medidas sobre la situación inflacionaria, pero que profundizarían su postura debido a que el 5 de abril de 1955 el consejo de ministros de Chile decidió contratar una comisión de técnicos para solucionar los problemas económicos y, pocos días después de ello, el 8 de abril, Julius Klein informó que el gobierno chileno se contactó con él para negociar la posibilidad de firmar un convenio con su consultora, decidiendo enviar a su socio, Julien Saks, a Santiago para evaluar la situación y negociar8 (Foreign Relations of the United States..., Documento 379, 13 de abril de 1955). A partir de esta situación, se delineó un plan de acción que

8 Dicho informe dice al respecto: "It has been and remains our policy to take advantage of every feasible opportunity to encourage the Chileans to take remedial action. It is possible that such an opportunity may be presented if the Chilean Council of Ministers carries out a proposal adopted on April 5 to employ a commission of technicians in economic and financial matters. Dr. Julius Klein stated on April 8 that he had been approached by the Chileans regarding the possibility of his firm's advising them on various administrative matters and that he planned to send his partner, Mr. Saks, to see what could be done”. Se puede encontrar en: https://history. state.gov/historicaldocuments/frus1955-57v07/d379. 
consistió en que: el Embajador estadounidense en Santiago debía incentivar y convencer a las autoridades chilenas para que adquirieran los servicios de una comisión de expertos para solucionar las dificultades inflacionarias ${ }^{9}$, que no se debía apoyar directamente a un grupo de técnicos en específico (esto con el propósito de evitar que se apuntara a Estados Unidos como responsable de la recomendación) ${ }^{10}$, que el Departamento de Estado y del Tesoro, el BIRF, el FMI y el Eximbank debían apoyar a Chile si les pedían ayuda en la contratación de una misión de expertos ${ }^{11}$ (Foreign Relations of the United States..., Documento 379, 13 de abril de 1955).

Y en una reunión del Operations Coordinating Board del mismo 13 de abril de 1955, donde se discutió la situación de Chile, se llegó al consenso que sería una buena medida que Ibáñez adquiriera los servicios de una consultora internacional como la Klein-Saks ${ }^{12}$ (Foreign Relations of the United States..., Documento 378, 13 de abril de 1955). Luego, el 19 de abril, desde el Departamento de Estado se informó a la Embajada estadounidense en Santiago que debían discretamente convencer a las autoridades chilenas de contratar una comisión de expertos de reputación, pero que tuvieran cuidado en no entregar directamente alguna recomendación ${ }^{13}$ (Foreign Relations of the United States..., Documento 380, 13 de abril de 1955).

La postura de presionar a Chile para que realizara reformas económicas y recibiera consejos de expertos extranjeros, continuó a través de las organizaciones financieras internacionales. Esto se observa en un informe enviado por la Embajada de Chile en Washington al Ministerio de Relaciones Exteriores, fechado para el 31 de mayo de 1955, con un relato sobre el viaje que realizó Oscar Herrera Palacios (Ministro de Educación en ese momento y desde octubre de 1955 sería Ministro de Hacienda) a Estados Unidos entre el 12 al 31 de mayo de 1955, donde tuvo una serie de reuniones con personeros de las instituciones financieras internacionales y del gobierno norteamericano. Entre las diferentes actividades y encuentros en que participó, para el día 24 de mayo a las 10:00 AM, se reunió con el presidente del Fondo Monetario Internacional, Ivar Rooth. Este último manifestó la necesidad de contratar expertos y aplicara las medidas necesarias:

9 Al respecto, dicho informe dice: "Through Embassy Santiago discreetly encourage the Chilean authorities to follow through on their announced intention of contracting for a commission".

10 Sobre este punto: "In carrying out the foregoing actions, refrain from sponsoring or appearing to sponsor a commission, so that the United States will not be regarded as responsible for its recommendations".

11 Al respecto: "Through State and Treasury, with the collaboration as appropriate of the International Bank (IBRD), the Export-Import Bank and the International Monetary Fund, aid Chile if requested to obtain the best available persons to serve on a commission".

12 El acta de dicha reunión establece que: "There was general agreement that it would be very worthwhile if the Chilean Government would obtain the services of a top-flight firm, such as Klein and Saks from New York." Ver: https://history.state.gov/historicaldocuments/frus1955-57v07/d378.

13 Dicho mensaje dice: "OCB has concurred in Department recommendation US through Embassy Santiago discreetly encourage Chilean authorities to follow through their announced intention of contracting commission and insure that Chileans select persons of ability integrity and reputation so that commission's findings will carry weight. In carrying out these actions Embassy should bear in mind US desire refrain from appearing to sponsor commission. FYI [For your information] Chileans have approached firm of Klein \& Saks and Saks planning visit Chile to discuss matter". Ver: https://history.state.gov/historicaldocuments/frus1955-57v07/d380. 
El señor Rooth expresó que, a su criterio, el principal problema de Chile era la inflación y que antes de que el Gobierno tomase medidas concretas y permanentes para su control, no era posible pensar en una ayuda exterior de importancia. (...) Comentó que, a su juicio, el problema chileno era lo suficientemente serio como para requerir una asistencia económica y financiera tanto de las instituciones internacionales especializadas, como del Eximbank e incluso del Gobierno de los Estados Unidos. Sin embargo, había que conocer previamente cuál era la magnitud del problema y contar, al mismo tiempo, con la decisión de aplicar todas aquellas medidas indispensables para controlar la inflación. Acentuó que esas medidas deberán ser previas a la ayuda internacional. (AMRREE, Vol. 4077, Confidencial N615-54, 31 de mayo de 1955, p. 3).

El mismo 24 de mayo, a las 11:00 AM, Herrera se entrevistó con el presidente del Banco Internacional de Reconstrucción y Fomento, Eugene Black. Este último se refirió también a la situación de la inflación y la posibilidad de aplicar un plan de desarrollo agrícola en Chile, planteando que dicho Plan de Desarrollo Agrícola y de los Transportes “era muy interesante", pero que no podía resolverse ni aplicarse nada al respecto "mientras no existiese una acción programada y parmente para controlar los factores inflacionistas". (AMRREE, Vol. 4077, Confidencial N615-54, 31 de mayo de 1955, p. 4). Además, Black habló de la contratación de la Misión Klein-Saks y a una situación de desencuentro que sucedió entre el BIRF y el gobierno chileno:

Dijo que estaba sorprendido de que se habría solicitado al Banco Internacional que seleccionara el experto más adecuado y, al mismo tiempo, de manera paralela, se había negociado y llegado a un acuerdo con una firma privada (Klein y Saks). Que una vez impuesto el Banco de esto último, no había estimado conveniente seguir adelante en sus gestiones. Expresó que Chile era -como es lógico- absolutamente libre para contratar un experto particular, al margen del Banco Internacional o del Fondo Monetario, pero que se permitía dar a conocer su opinión personal, en el sentido de que si Chile es miembro de estas dos instituciones; mantiene relaciones de crédito con ellas; precisa utilizarlas en el futuro y tiene asuntos en estudio de tanta trascendencia como el Plan Agrícola, parece aconsejable recibir su opinión en materias económicas y financieras. Declaró que tenía un alto concepto del señor Julius Klein y que sabía que la firma Klein y Saks era de prestigio, pero que el Banco Internacional, por razones más bien de orden técnico, no la contrataría para atender asuntos de su jurisdicción. Expresó que comprendía que muy distinta podía ser la posición de un Gobierno. (AMRREE, Vol. 4077, Confidencial N615-54, 31 de mayo de 1955, p. 4).

Si bien las palabras de Black expresan una cierta disconformidad por la elección de la firma Klein-Saks, finalmente agregó que, independiente de los expertos que se escogieran, lo importante era que fuesen eficientes en establecer cuáles eran los problemas de Chile y las 
medidas necesarias para su solución (AMRREE, Vol. 4077, Confidencial Nº15-54, 31 de mayo de 1955, p. 4).

En el mismo día 24 de mayo, a las 03:00 PM, Herrera se reunió con miembros del Directorio del Banco de Exportaciones e Importaciones, instancia en la cual no hubo comentarios sobre la Misión Klein-Saks, pero sí plantearon los representantes del Eximbank que el principal problema de Chile era la inflación (AMRREE, Vol. 4077, Confidencial N61554, 31 de mayo de 1955, p. 5).

El día 25 de mayo, a las 10:30 AM, Herrera se reunió con el Secretario Adjunto del Departamento del Tesoro, Andrew Overby, quien le planteó que lo más urgente de la situación chilena era combatir el alza de los precios y que desde Estados Unidos se “(...) esperaba que Chile precisara un programa de acción decidida contra la inflación, a fin de poder estudiar alguna ayuda que no se perdiera". (AMRREE, Vol. 4077, Confidencial N615-54, 31 de mayo de 1955, p. 6). Además, se refirió al convenio con la Misión Klein-Saks, aludiendo a la necesidad de una comisión de expertos, pero dejando en claro que Chile debía tomar la decisión sobre a quiénes contratar:

Dijo [Overby] que como funcionario del Gobierno no podía opinar sobre los méritos de esta o aquella firma. Que si se hablaba de los señores Klein \& Saks, estaba en situación personal de declarar que conocía al señor Klein desde hace años y que tenía un alto concepto de él y de su pericia técnica, ya demostrada en otros países. Conocía igualmente al señor Saks, pero no tanto como al señor Klein. Sin embargo, el problema es otro: ¿conviene al Gobierno de Chile el consejo de una forma privada de expertos o el de una Misión seleccionada a base de la recomendación del Banco Internacional $o$ del Fondo Monetario, o de ambos? Manifestó que Chile, como miembro del Banco y del Fondo, tenía experiencia con esas instituciones, las que, a su turno, conocían los problemas de Chile. Que el Banco siempre había seleccionado misiones idóneas en sus estudios de otros países; que esas misiones eran integradas con expertos de cada uno de los aspectos básicos de una economía, como ser, producción agrícola, minera, industrial o transportes, etc. Que el Fondo Monetario era la institución dedicada exclusivamente a problemas financieros. Luego, si el Gobierno de Chile deseaba una Misión Económica, era recomendable conversarlo con el Banco, si quería una Misión Financiera, con el Fondo Monetario. En conclusión, aun cuando él no podía decir qué sería lo más apropiado, se permitía indicar que el Gobierno de Chile debería decir sobre qué tipo de consejo o asesoría precisa, y según sea la respuesta que se dé, elegir la misión con participación del Banco Internacional o del Fondo Monetario, o recurrir al sector privado. (AMRREE, Vol. 4077, Confidencial N615-54, 31 de mayo de 1955, p. 7).

De todas estas reuniones sostenidas por Herrera, se constata que, tanto los estadounidenses como las instituciones económicas y financieras internacionales, incitaron 
al gobierno chileno para que recibiera recomendaciones de expertos para solucionar el problema de la inflación. También, se puede pensar que condicionaron la entrega de ayuda económica a Chile, obligado al gobierno de Ibáñez a elaborar un plan destinado a solucionar los problemas económicos, el que debía ser aprobado por las autoridades estadounidenses y de las instituciones financieras internacionales, lo cual se puede ver en la reunión que sostuvo Herrera con el FMI, con el BIRF y con el Departamento del Tesoro.

Además, destacar que, a partir de la revisión de las fuentes, no se aprecia un apoyo directo a la contratación de la Misión Klein-Saks de parte de las autoridades estadounidenses o de los organismos internacionales. Ello fue una postura presente en el plan de acción que establecieron desde el gobierno norteamericano. Y, aún más, se expresó la intención de postular a los técnicos del FMI y del BIRF como opciones posibles para realizar la tarea de elaborar un plan de estabilización de la economía en Chile, aunque dejaron en claro que la decisión final la tenía el propio gobierno de Ibáñez.

Finalmente, el 27 de junio de 1955 se ordenó a la Embajada chilena en Washington desde el Ministerio de Relaciones Exteriores que iniciaran las gestiones para la firma definitiva del contrato entre Chile y la consultora Klein-Saks (AMRREE, Vol. 4077, Confidencial N ${ }^{\circ} 857-62$, 22 de julio de 1955 , p. 1).

\section{CONCLUSIONES}

En este escrito se examinó la participación que tuvieron el gobierno estadounidense y los organismos financieros internacionales en el proceso que llevó a la contratación de la Misión Klein-Saks por parte de Chile en 1955. Un primer elemento a considerar y que ayuda a entender el fenómeno, es el contexto de la Guerra Fría, el cual enmarcó o limitó el rango de acción internacional durante el periodo. Desde el punto de vista estadounidense, para evitar una "infiltración comunista" y resguardar la seguridad nacional, se interesaron en que los países del Tercer Mundo y dentro de su órbita de influencia, como Chile, tuvieran estabilidad y un mejoramiento de las condiciones de vida de su población. Desde la perspectiva chilena, dependían bastante del comercio con Estados Unidos y de la ayuda económica recibida de esa nación, lo cual era una limitante importante ante la eventualidad de tomar un rumbo de acción que significara ir en contra de los norteamericanos.

Un segundo elemento a considerar es el contexto interno de Chile durante los primeros años del gobierno de Ibáñez, caracterizado por una agudización de la inflación y un complejo panorama político. Si bien el problema del alza de los precios no era nuevo, a partir de 1953 comenzó un aumento importante y, ante ello, era necesario buscar solución a los problemas económicos. El gobierno de turno intentó aplicar medidas al respecto, llegando finalmente a la contratación de la Misión Klein-Saks, que comenzó su trabajo en septiembre de 1955.

Tercero, revisando las fuentes diplomáticas chilenas y estadounidenses, se observa que hubo un interés por parte del gobierno de EE.UU. que se aplicaran nuevas medidas en Chile 
para estabilizar la situación macroeconómica, y establecieron una estrategia de incentivar y presionar, mediante la diplomacia y los organismos financieros internacionales (FMI, BIRF y Eximbank), al gobierno chileno para que solucionaran la alta inflación, al menos desde fines de 1954. Ya para marzo-abril de 1955, la postura estadounidense y de los organismos internacionales fue de plantearle a Chile la necesidad de adquirir los servicios de expertos que ayudasen a elaborar un plan de estabilización económica. A esto hay que agregar que no recomendaron directamente a la firma Klein-Saks, más bien solo presionaron para la contratación de expertos y, al momento en que informaron la contratación de la Misión KleinSaks, los estadounidenses se mostraron conformes con ello.

Teniendo estos tres niveles de análisis, se puede comentar que: la estrategia de EE.UU. de presionar a Chile para que estabilizara la inflación se enmarcó dentro de la política de la Guerra Fría de ayudar al desarrollo de los países del Tercer Mundo para evitar la infiltración comunista. También, que posiblemente era difícil para Chile desentenderse de la postura estadounidense y la opción más realista era ceder a sus presiones, teniendo en consideración los vínculos comerciales entre ambos países y los dineros internacionales que recibía Chile de las instituciones internacionales. A esto se agrega que, durante el gobierno de Ibáñez, la situación económica fue compleja y desde los organismos financieros internacionales incluso deslizaron la idea de condicionar la ayuda internacional a Chile si no solucionaba el problema inflacionario, haciendo aún más difícil para la administración de turno desentenderse de las palabras de los estadounidenses.

En el proceso de contratación de la Misión Klein-Saks, las presiones estadounidenses y de los organismos financieros internacionales fueron un factor que efectivamente existió entre fines de 1954 y 1955. Si ello fue una variable decisiva, faltan mayores antecedentes para afirmarlo. Sin embargo, considerando vínculos económicos de Chile con EE.UU. y el interés del gobierno de Ibáñez por adquirir préstamos internacionales, se puede pensar que posiblemente fue un factor que tuvieron en consideración al momento de buscar el asesoramiento de expertos extranjeros.

\section{REFERENCIAS}

Collier, S. \& Sater, W. (1999). Historia de Chile: 1908-1994. Madrid: Cambridge University Press.

Correa, S. (1985). Algunos antecedentes históricos del proyecto neoliberal en Chile (19551958). OPCIONES, 6, 106-146.

Correa, S., Figueroa, M., Jocelyn-Holt, A., Rolle, C., \& Vicuña, M. (2001). Historia del siglo XX chileno. Balance paradojal. Santiago de Chile: Editorial Sudamericana.

Couyoumdjian, J. (2011). ¿Por qué la Misión Klein-Saks? Los orígenes de un programa de estabilización económica para Chile. En J. Couyoumdjian (Ed.), Reformas económicas e instituciones políticas: la experiencia de la Misión Klein-Saks en Chile (pp. 61-101). 
Santiago de Chile: Universidad del Desarrollo.

Edwards, S. (2011). Estableciendo credibilidad: el rol de los consultores extranjeros en el programa de estabilización de 1955-1958 en Chile. En J. Couyoumdjian (Ed.), Reformas económicas e instituciones políticas: la experiencia de la Misión Klein-Saks en Chile (pp. 103-164). Santiago de Chile: Universidad del Desarrollo.

Fermandois, J. (2002). El sistema CORFO y la época del "subsidio" en Chile, 1939-1973. Bicentenario, 2(1), 23-52.

Fermandois, J. (2005). Mundo y fin del Mundo. Chile en la política mundial 1900-2004. Santiago de Chile: Ediciones Universidad Católica de Chile.

Fermandois, J. (2009). La internacionalización de la historia internacional. En F. Purcell \& A. Riquelme (Eds.), Ampliado miradas. Chile y su historia en un tiempo global (pp. 25-41). Santiago de Chile: RIL editores.

Fermandois, J., Bustos, J., \& Schneuer, M. (2009). Historia política del cobre 1945-2008. Santiago de Chile: Centro de Estudios Bicentenario.

Ffrench-Davis, R. (1973). Políticas económicas en Chile, 1952-1970. Santiago de Chile: Centro de Estudios de Planificación Nacional.

Gil, F. (1969). El sistema político en Chile. Santiago de Chile: Editorial Andrés Bello.

Hirschman, A. (1964). Estudios sobre política económica en América Latina (en ruta hacia el progreso). Madrid: Aguilar.

Hobsbawm, E. (1999). Historia de siglo XX. Bueno Aires: Crítica.

Hove, M. (2009). Losing a model democracy: Salvador Allende and U.S.-Chilean relations, 19451970 (Tesis doctoral). Recuperada de: http://ufdc.ufl.edu/UFE0024361/00001.

Kofas, J. (1999). Stabilization and class conflict: The State Department, the IMF, and the IBRD in Chile, 1952-1958. The International History Review, 2(21), 352-385.

Moulian, T. (1986). El Gobierno de Ibáñez, 1952-1958. Santiago de Chile: Programa FlacsoSantiago de Chile.

Muñoz, H., \& Portales, C. (1987). Una amistad esquiva: las relaciones de Estados Unidos y Chile. Santiago de Chile: Pehuén Editores.

Pereira, J., \& Neila, J. (2003). La Historia de las Relaciones Internacionales como disciplina científica. En J. Pereira (Coord.), Historia de las relaciones internacionales contemporáneas (pp. 13-36). Barcelona: Editorial Ariel.

Pinto. A. (1964). Chile: una economía difícil. Ciudad de México: Fondo de Cultura Económica.

Sater, W. (1990). Chile and the United States: Empires in Conflict. London: The University of Georgia Press.

Sierra, E., Benavente, S., \& Osorio J. (1967). Las políticas de estabilización de Chile en el periodo 1956-66. Tomo I. Segunda parte. La política de 1956-58. (Tesis inédita para optar al título de Ingeniero Comercial). Universidad de Concepción. Concepción, Chile. Ms.

Smith, P. (1996). Talons of the Eagle. Dynamics of U.S.-Latin American relations. New York: Oxford University Press. 
Wilhelmy, M. (1979). Hacia un análisis de la política exterior chilena contemporánea. Estudios Internacionales, 12(48), 440-471.

Würth, E. (1958). Ibáñez caudillo enigmático. Santiago de Chile: Editorial Pacífico.

Y [Louis Halle]. (1950). On a certain impatience with Latin America. Foreign Affairs, 28(4), 576-576. Recuperado de: http://americanempireproject.com/ empiresworkshop/chapter1/TheTwentiethCentury-CompletingTheRevolution/ LouisHalles1950YArticleOnLatinAmerica.pdf.

\section{Fuentes}

Archivo Histórico del Ministerio de Relaciones Exteriores (AMRREE), Fondo Histórico. Volúmenes 4639, 4077.

Foreign Relations of the United States, 1952-1954, American Republics, Volume IV, Political and Economic Relations of the United States and Chile. https://history.state.gov/ historicaldocuments/frus1952-54v04/ch10. Documento: 260.

Foreign Relations of the United States, 1955-1957, American Republics: Central and South America, Volume VII, Political and Economic Relations of the United States and Chile. https://history.state.gov/historicaldocuments/frus1955-57v07/ch11. Documentos: $378,379,380$. 